\title{
VALUE DISTRIBUTION OF HARMONIC AND FINELY HARMONIC MORPHISMS AND APPLICATIONS IN COMPLEX ANALYSIS
}

\author{
BENT FUGLEDE
}

\section{Introduction}

The aim of this article is to generalize (e.g. to several complex variables) and in part to strengthen the following three classical results on removable singularities of meromorphic functions in which either the singularity set or the corresponding cluster set is polar, i.e., locally of zero outer (logarithmic) capacity.

Let $U$ denote a domain in $\mathbf{C}$ (or on a Riemann surface), and $F$ a relatively closed proper subset of $U$. A meromorphic function $\varphi: U \backslash F \mapsto \mathbf{C}_{\infty}(=\mathbf{C} \cup\{\infty\})$ extends then uniquely to a meromorphic function on all of $U$ in each of the following 3 situations (of which a) is subsumed in b) here, but not always in our generalizations]:

a) $F$ is polar, and the cluster set $C(\varphi, z)$ is distinct from $\mathbf{C}_{\infty}$ for every $z \in F$.

b) $F$ is polar, and every $z \in F$ has a neighbourhood $W$ in $U$ such that $\mathbf{C}_{\infty} \backslash \varphi(W \backslash F)$ is non-polar.

c) $C(\varphi, F)$ is polar (in $\mathbf{C}_{\infty}$ ), and $\varphi^{\prime} \not \equiv 0$ in $U \backslash F$. (It follows non-trivially that $F$ itself is likewise polar.)

Here $C(\varphi, F)$ denotes the cluster set of $\varphi$ at $F$, that is the union of all $C(\varphi, z)$ as $z$ ranges over the boundary of $F$ relative to $U$.

Our strengthening of the results a) and c) consists essentially in replacing the cluster sets by the corresponding (a priori smaller) fine cluster sets. This means that the standard topology on $\mathbf{C}$ for the independent variable is replaced by the Cartan fine topology (the weakest topology making all subharmonic functions continuous). - For a survey of the fine topology and some of its applications see [34].

The above result in the situation a) amounts to (closed) polar sets being removable singularity sets for bounded holomorphic functions. And this, in turn, derives from the removability of polar singularity sets for bounded harmonic functions, a result going back to Bouligand [7] (1926) and Myrberg [58] (1933). They also showed that (compact) polar sets are the only removable singularity sets for bounded harmonic functions. This contrasts the situation for bounded holomorphic functions where the compact sets of zero analytic capacity form the precise class of (compact) removable singularity sets, see e.g. Garnett [37]. In particular, the com- 
pact sets of 1-dimensional Hausdorff measure 0 form a class (containing amply the class of compact polar sets) of removable singularity sets for bounded holomorphic functions, as shown by Painlevé, see also Zoretti [81] (1905) and Besicovich [4].

In the case where $F$ reduces to a single point, a) is the classical CasoratiWeierstrass theorem (see [13, note on p. 4] for historical comments). It was strengthened by Doob [23] in 1965 who replaced the cluster set by the fine cluster set (in the case where $F$ is a singleton). Doob suggests (in the same paper) that one should see what, if any, contribution the fine topology has to offer to the extensive theory of cluster values of meromorphic functions.

The result in the situation b) is due to Nevanlinna [61, Kap. V, $\S 4$, Satz 3] (1936) for $U=\mathbf{C}_{\infty}$, and to Kametani [48] (1941) for any $U$ (both for compact $F$ only). See also the monographs [79], [62], [13].

The result in the situation c) is Radó's theorem, essentially as established in [72] (1924) in the case where $C(\varphi, F)=\{0\}$. In that case the result asserts that a continuous function $\varphi: U \rightarrow \mathbf{C}$ is holomorphic in $U$ if it is holomorphic in $U \backslash \varphi^{-1}(0)$. This was extended by Cartan [10] (1952) to holomorphic functions of several variables. And this $n$-dimensional version was further extended by Lelong [54] (1957) who replaced $\varphi^{-1}(0)$ by $\varphi^{-1}(E)$ for any closed polar set $E \subset \mathbf{C}$. The still more general cluster set version (now again in one complex variable, essentially as stated in c) above) is due to Stout [77] (1968). Further extensions were given by Goldstein and Chow [39], Järvi [47], Boboc [5], Cole and Glicksberg [12], Cegrell [11], Oja [65], Riihentaus [73], and Øksendal [66]. - The interesting question raised by Stout [77] whether the polarity of $E$ above (respectively of $C(\varphi, F)$ ) can be replaced by some weaker hypothesis seems to remain open.

The Radó type results obtained in the present paper (notably Theorems 2, 4, 6, 7 and 8) can be regarded as generalizations of almost all the results of this type quoted above. The starting point is Cartan's simple proof [10] of the original Radó theorem, based on the subharmonicity of $\log |\varphi|$ in all of $U$ when $\varphi$ is non-constant and continuous in $U$ and holomorphic in $U \backslash \varphi^{-1}(0)$. This allowed Cartan to reduce Radó's theorem to the removability of closed polar sets as singularity sets for bounded holomorphic functions, that is, the result a) stated above. As noted by Aupetit [1], the same method carries over to give a simple proof of Stout's cluster set version of Radó's theorem stated in c) above.

Briefly speaking, the idea in Cartan's proof of Radó's theorem rests on the following potential theoretic property of a holomorphic function $\varphi$ defined in a domain in $\mathbf{C}^{n}: \varphi$ is continuous, and $u \circ \varphi$ is harmonic in $\varphi^{-1}(V)$ for any harmonic function $u$ in a domain $V$ in $\mathbf{C}$. This type of mapping, generalizing holomorphic functions, can be traced back to Jacobi [46] in 1848 (who considered mappings from $\mathbf{R}^{3}$ into $\mathbf{C}$ ). Continuous mappings with the stated property of preservation of harmonicity are now called harmonic morphisms (or harmonic maps). In more recent time harmonic morphisms have been studied by Gehring and Haahti [38] 
(1960), by Constantinescu and Cornea [17] (1965), and by Sibony [76] (1968). Further contributions were made by Hansen [41], Meghea [57], Ikegami [43], [44], Laine [50], [52], Schirmeier [74], [75], Boboc [5], Fuglede [29], [30], [31], [32], Ishihara [45], Bernard, Campbell, and Davie [3], Oja [63], [64], [65], Baird and Eells [2], Pirinen [70], Øksendal and Csink [19], and Popa [71]. (This list is not complete.)

The quoted paper of Boboc [5] (1978) seems to be the first in which the RadóStout theorem is extended to harmonic morphisms. In the same spirit we shall in the present paper extend the above results a), b), c) to harmonic morphisms between suitable harmonic spaces $X$ and $X^{\prime}$. At the expense of slightly reducing the generality of these spaces (notably by imposing on $X$ the axiom of domination, rather than just ellipticity as in [5]) we choose to perform these extensions first in the more general frame of finely harmonic morphisms (Section 2), that is, the generalization of harmonic morphisms to mappings from a finely open subset of $X$ into $X^{\prime}$. This is a natural general frame for our use of fine cluster sets. By specialization afterwards to usual harmonic morphisms (Section 4) and to complex analysis in one dimension (Section 3) or in several dimensions (Section 5) we arrive at the desired extended and in part stronger forms of the classical results a), b), and c) above. We also obtain analogues (not extensions) of the Riesz-Frostman-Nevanlinna-Tsuji theorem on boundary cluster sets of a meromorphic function on the disc.

Finely harmonic morphisms were studied by Laine [51], [53], Fuglede [28], [32], Øksendal [67], and Popa [71].

An account of the results of the present paper (without proofs) was given in [35].

I wish to thank T. Ikegami, J. Král, I. Laine, B. Øksendal, M. Shiba, and $\mathrm{H}$. Tornehave for valuable information.

\section{Notations and preliminaries}

In Sections 1, 2, and 4, $X$ and $X^{\prime}$ denote two harmonic spaces in the sense of [18] with a countable base for their topology. Except when otherwise stated it is further supposed that $X$ satisfies the axiom of domination (Axiom D), and that $X^{\prime}$ is weakly $\mathfrak{P}$-harmonic (cf. Definition 1 below). These basic hypotheses will not be repeated. They are satisfied e.g. if $X$ and $X^{\prime}$ are Riemannian manifolds (each endowed with the sheaf of solutions to the Laplace-Beltrami equation). Applications to complex analysis are given in Sections 3 and 5.

There would be no actual loss of generality in assuming $X$ to be connected. Similarly as to $X^{\prime}$, except perhaps in results of the type of Radó's theorem.

Recall that a harmonic space (with countable base) is a $\mathfrak{P}$-harmonic space if and only if it admits a potential $>0$, cf. [18, Proposition 7.2.1]. An open subset of a harmonic space is called a $\mathfrak{B}$-set if it is $\mathfrak{P}$-harmonic as a harmonic subspace. Every union of pairwise disjoint $\mathfrak{P}$-sets of a harmonic space is a $\mathfrak{P}$-set. Every harmonic 
space admits a covering by $\mathfrak{P}$-sets [18, Theorem 2.3.3]. A Riemannian manifold is a $\mathfrak{P}$-harmonic space if and only if it has a Green function.

A subset $E$ of a harmonic space is called polar if locally (hence actually on each $\mathfrak{P}$-set) there exists a superharmonic function $\geqq 0$ equal to $+\infty$ in $E$, cf. [18, Prop. 6.2.1 and Exerc. 6.2.1].

Definition 1. A harmonic space $X^{\prime}$ will be called weakly $\mathfrak{P}$-harmonic if any open subset $W^{\prime}$ of a component $Y^{\prime}$ of $X^{\prime}$ is a $\mathfrak{P}$-set provided that $Y^{\prime} \backslash W^{\prime}$ is nonpolar (thus in particular when $Y^{\prime}$ is non-compact and $W^{\prime}$ is relatively compact in $Y^{\prime}$ ).

By a theorem which goes back to Szegő [78] and Myrberg [58], every elliptic ङ-harmonic space is weakly $\mathfrak{P}$-harmonic, cf. Constantinescu and Cornea [15], [18, Exerc. 6.2.5]. In particular, every Riemannian manifold is a weakly $\mathfrak{P}$-harmonic space, being a Brelot space (hence elliptic) admitting a superharmonic function $>0$ (e.g. the constant 1 which is harmonic).

On the first harmonic space $X$ we shall primarily use the fine topology, but sometimes also the usual (=initial) topology. Qualifications pertaining to the fine topology are indicated by "fine(ly)". The fine boundary of a set $F \subset X$ is denoted by $\partial_{f} F$. Recall that every polar set $F$ is finely closed and finely isolated and has no finely interior points, cf. [18, Proposition 6.2.3], whence $\partial_{f} F=F$.

On the second harmonic space $X^{\prime}$ we shall always employ the usual topology. The closure $\bar{A}^{\prime}$ of a subset $A^{\prime}$ of $X^{\prime}$ is always taken in the one-point compactification $X_{\infty}^{\prime}\left(=X^{\prime} \cup\{\infty\}\right.$ or $\left.X^{\prime}\right)$ of the locally compact (possibly compact) space $X^{\prime}$. (Any other compactification of $X^{\prime}$ could be used instead with exactly the same results.)

Since the fine topology is generally not even 1 st countable, filters must be used in the definition of fine cluster sets.

Definition 2. For any mapping $\varphi$ of a finely open set $V \subset X$ into $X^{\prime}$ and for any $x \in \partial_{f} V$ the fine cluster set $C_{f}(\varphi, x)$ of $\varphi$ at $x$ is defined as the set of all points $x^{\prime} \in X_{\infty}^{\prime}$ for which there exists a filter $\mathfrak{F}$ on $V$ converging finely to $x$ such that $\varphi(F) \rightarrow x^{\prime}$ in $X_{\infty}^{\prime}$. Equivalently,

$$
C_{f}(\varphi, x)=\cap \overline{\varphi(W \cap V)}
$$

as $W$ ranges over a fundamental system of fine neighbourhoods of $x$ in $X$.

Since $X_{\infty}^{\prime}$ is compact, $C_{f}(\varphi, x)$ is a non-empty compact subset of $X_{\infty}^{\prime} \cdot$ Moreover, for any open set $W^{\prime} \subset X_{\infty}^{\prime}$ such that $C_{f}(\varphi, x) \subset W^{\prime}$ there is a fine neighbourhood $W$ of $x$ in $X$ such that $\varphi(W \cap V) \subset W^{\prime}$. In particular, $C_{f}(\varphi, x)$ reduces to a single point $x^{\prime}$ if and only if

$$
\text { fine } \lim _{y \rightarrow x} \varphi(y)=x^{\prime}
$$

If $\varphi: V \rightarrow X^{\prime}$ is finely continuous (i.e., continuous with respect to the fine 
topology on $X$ and the usual topology on $X^{\prime}$ ) and if $X \backslash V$ is thin at $x$ (that is, if $x$ is a polar and finely isolated point of $[V)$, then $C_{f}(\varphi, x)$ is connected. (This follows from the local connectedness of the fine topology on $X$ together with the fact that if $W$ is a fine domain in $X$ and $E$ a polar subset of $W$ then $W \backslash E$ is finely connected, see [25], [26, Theorem 12.2].) - There is a similar observation in which the usual topology is employed throughout in place of the fine topology.

Recall that, for a usual open set $U \subset X$, the fine components of $U$ are the same as the usual components of $U$, see [25], [26, §9.8].

Definition 3. For any mapping $\varphi$ of a finely open set $V \subset X$ into $X^{\prime}$ and for any set $F \subset X$ not meeting $V$ the fine cluster set of $\varphi$ at $F$ (more precisely at $F \cap \partial_{f} V$ ) is defined as

$$
C_{f}(\varphi, F)=C_{f}\left(\varphi, F \cap \partial_{f} V\right)=\bigcup_{x \in F \cap \partial_{f} V} C_{f}(\varphi, x) .
$$

For a mapping $\varphi$ of a usual open set $V \subset X$ into $X^{\prime}$ and for any point $x \in \partial V$, respectively any set $F \subset X \backslash V$, the usual cluster sets $C(\varphi, x)$ and $C(\varphi, F)$ are defined in analogy with the above definitions, replacing throughout the fine topology on $X$ by the usual topology.

\section{Finely harmonic morphisms}

Definition 4. A finely continuous mapping $\varphi$ of a finely open set $U \subset X$ into $X^{\prime}$ is called a finely harmonic morphism if $s^{\prime} \circ \varphi$ is finely hyperharmonic in $\varphi^{-1}\left(V^{\prime}\right)$ for every (usual) hyperharmonic function $s^{\prime}$ in an open set $V^{\prime} \subset X^{\prime}$.

If $X^{\prime}$ has a base (for the usual topology) formed by regular sets then this definition is equivalent to the original one in [28] in which $s^{\prime}$ was required to be harmonic in $V^{\prime}$ and $s^{\prime} \circ \varphi$ finely harmonic in $\varphi^{-1}\left(V^{\prime}\right)$, cf. [32, § 2.3]. (In [28], $X^{\prime}$ was supposed to satisfy Axiom $D$ and hence to have a base of regular sets, cf. [18, p. 233].)

In the very particular case where $X^{\prime}=\mathbf{R}$ (endowed with the affine sheaf) the finely harmonic morphisms $U \rightarrow \mathbf{R}$ are nothing but the finely harmonic functions on $U$.

In the following proposition the general hypothesis that $X^{\prime}$ be weakly $\mathfrak{P}$-harmonic is not needed (and not used in the proof).

Proposition 1. Let $U$ denote a finely open subset of $X$, and $F$ a polar set contained in $U$. Let $\varphi: U \rightarrow X^{\prime}$ be finely continuous and suppose that the restriction of $\varphi$ to $U \backslash F$ is a finely harmonic morphism of this set into $X^{\prime}$. Then $\varphi$ is a finely harmonic morphism of $U$ into $X^{\prime}$.

Proof. With $s^{\prime}$ and $V^{\prime}$ as in Definition 4 above, $s^{\prime} \circ \varphi$ is finely continuous in the finely open set $\varphi^{-1}\left(V^{\prime}\right)$ and finely hyperharmonic in $\varphi^{-1}\left(V^{\prime}\right) \backslash F$, hence finely hyperharmonic in all of $\varphi^{-1}\left(V^{\prime}\right)$ according to [26, Theorem 9.14]. 
We proceed to extend Proposition 1 to the situation where $\varphi$ is defined on $U \backslash F$ only, without assuming a priori that $\varphi$ should have a fine limit at the points of $F$. Since $F$ is polar, the fine cluster set $C_{f}(\varphi, x)$ at each point $x \in F$ is a compact connected subset of $X_{\infty}^{\prime}$ (Section 1).

Theorem 1. Let $U$ denote a finely open subset of $X$, and $F$ a polar set contained in $U$. A finely harmonic morphism $\varphi: U \backslash F \rightarrow X^{\prime}$ extends to a unique finely harmonic morphism of $U$ into $X^{\prime}$ if (and only if) the following two conditions are fulfilled:

i) $C_{f}(\varphi, F) \subset X^{\prime}$.

ii) For every $x \in F$ such that the component $Y^{\prime}$ of $X^{\prime}$ containing $C_{f}(\varphi, x)$ is compact and not a $\mathfrak{P}$-set, there is a fine neighbourhood $W$ of $x$ in $U$ such that $Y \backslash \varphi(W \backslash F)$ is non-polar.

Proof. The uniqueness is obvious since the polar set $F$ has no finely interior points. The "only if" part is likewise evident. In proving the "if" part it suffices to show that, for each $x \in F$, the fine cluster set $K^{\prime}:=C_{f}(\varphi, x)$ (contained in $X^{\prime}$ by i)) reduces to a single point, for then $\varphi$ extends to a finely continuous mapping $\varphi^{*}: U \rightarrow X^{\prime}$ to which Proposition 1 applies.

Let $Y^{\prime}$ denote the component of $X^{\prime}$ containing $K^{\prime}$, and consider first the case where $Y^{\prime}$ is a $\mathfrak{P}$-set. Choose a finely open set $W \subset U$ containing $x$ and such that $\varphi(W \backslash F) \subset Y^{\prime}$. Proceeding as in the proof in [5] we choose $a^{\prime}, b^{\prime} \in K^{\prime}$ and filters $\mathfrak{F}, \mathfrak{G}$ on $W \backslash F$ converging finely to $x$ such that $\varphi(\mathfrak{F}) \rightarrow a^{\prime}, \varphi(\mathfrak{F}) \rightarrow b^{\prime}$. For any continuous hyperharmonic function $s^{\prime} \geqq 0$ on $Y^{\prime}, s^{\prime} \circ \varphi$ is finely hyperharmonic and $\geqq 0$ on $W \backslash F$ because $\varphi$ is a finely harmonic morphism on $U \backslash F$. Since $F$ is polar, $s^{\prime} \circ \varphi$ extends by fine continuity to a finely hyperharmonic function $s$ on $W$ according to [26, Theorem 9.14], and hence $\left(s^{\prime} \circ \varphi\right)(\mathfrak{F}) \rightarrow s(x)$. On the other hand, $\left(s^{\prime} \circ \varphi\right)(\mathscr{F}) \rightarrow s^{\prime}\left(a^{\prime}\right)$ by the continuity of $s^{\prime}$. It follows that $s^{\prime}\left(a^{\prime}\right)=s(x)$, and similarly $s^{\prime}\left(b^{\prime}\right)=s(x)$. Since the functions $s^{\prime}$ as above separate the points of $Y^{\prime}$ (even linearly), cf. [18, Proposition 2.3.2], we conclude that $a^{\prime}=b^{\prime}$, showing that $K^{\prime}$ is indeed a singleton.

In the case where $Y^{\prime}$ is non-compact we choose a relatively compact, open set $W^{\prime}$ so that $K^{\prime} \subset W^{\prime} \subset Y^{\prime}$. Proceeding as above, now with the $\mathfrak{P}$-set $W^{\prime}$ in place of $Y^{\prime}$, we see that $K^{\prime}$ is a singleton in this case too.

Finally suppose that $Y^{\prime}$ is compact and not a $\mathfrak{P}$-set. By hypothesis, there is a finely open set $W$ such that $x \in W \subset U$ and that $Y^{\wedge} \backslash \varphi(W \backslash F)$ is non-polar. We may suppose, moreover, that $\varphi(W \backslash F) \subset Y^{\prime}$. Finally we may arrange, by diminishing $W$ suitably, that $\varphi(W \backslash F)$ is a Borel set. In fact, every $y \in W \backslash F$ has a fine neighbourhood $K(y) \subset W \backslash F$, compact in the usual topology on $X$, and such that the restriction of $\varphi$ to $K(y)$ is continuous (with respect to the usual topologies on $K(y)$ and $Y^{\prime}$ ), cf. [28, Lemma 1]. By Doob's quasi Lindelöf principle, cf. [24], [18, Exerc. 7.2.6], there is a sequence $\left(y_{n}\right) \subset W \backslash F$ such that the sets $K\left(y_{n}\right)$ 
cover $W \backslash F$ up to a polar set $E$. The set $W \backslash E$ (to replace $W$ ) is then finely open and contains $x$, and

$$
\varphi(W \backslash E \backslash F)=\varphi\left(\bigcup_{n} K\left(y_{n}\right)\right)=\bigcup_{n} \varphi\left(K\left(y_{n}\right)\right)
$$

is a $K_{\sigma}$-set in $Y^{\prime}$. We may thus indeed assume that $\varphi(W \backslash F)$ is a Borel set contained in $Y^{\prime}$ and that $Y^{\backslash} \backslash \varphi(W \backslash F)$ is non-polar.

If $K^{\prime} \backslash \varphi(W \backslash F)$ contains non-polar points we fix one such point $c^{\prime}$. Let $a^{\prime}, b^{\prime} K^{\prime}$, and suppose that $a^{\prime} \neq c^{\prime}, b^{\prime} \neq c^{\prime}$ if $c^{\prime}$ exists. We propose to choose a P-set $W^{\prime} \subset Y^{\prime}$ containing $\varphi(W \backslash F) \cup\left\{a^{\prime}, b^{\prime}\right\}$. If $c^{\prime}$ above exists, it suffices to take $W^{\prime}=Y^{\prime} \backslash\left\{c^{\prime}\right\}$. Suppose therefore that $c^{\prime}$ does not exist. Then $a^{\prime}$ must be polar in case $a^{\prime} \notin \varphi(W \backslash F)$, and similarly as to $b^{\prime}$. It follows that the Borel set $Y^{\prime} \backslash\left(\varphi(W \backslash F) \cup\left\{a^{\prime}, b^{\prime}\right\}\right)$ is non-polar, hence contains a compact non-polar set $H^{\prime}$ according to an instance of Choquet's capacitability theorem, cf. [18, Proposition 6.2.2]. Now $W^{\prime}:=Y^{\prime} \backslash H^{\prime}$ is a $\Re$-set in $Y^{\prime}$ containing $\varphi(W \backslash F) \cup\left\{a^{\prime}, b^{\prime}\right\}$.

Proceeding as in the case where $Y^{\prime}$ is a $\mathfrak{P}$-set we find (replacing $Y^{\prime}$ by $W^{\prime}$ ) that $a^{\prime}=b^{\prime}$, and so $K^{\prime}$ reduces to two points or one point, viz. $K^{\prime}=\left\{a^{\prime}, c^{\prime}\right\},\left\{c^{\prime}\right\}$ or $\left\{a^{\prime}\right\}$ depending on whether or not $c^{\prime}$ exists. Being connected, $K^{\prime}$ is indeed a singleton.

We next bring a Radó-Lelong-Stout type result corresponding essentially to Theorem 1, except that now $C_{f}^{\prime}(\varphi, F)-\cdots$ rather than $F$ itself - is supposed to be polar.

Theorem 2. Let $U$ denote a fine domain in $X$, and $F$ a relatively finely closed proper subset of $U$. Let $\varphi$ be a finely harmonic morphism, not finely locally constant, of $U \backslash F$ into $X^{\prime}$. If $C_{f}(\varphi, F)$ is a polar subset of $X^{\prime}$, then $F$ is polar (in $X$ ), and $\varphi$ extends to a unique finely harmonic morphism of $U$ into $X^{\prime}$.

Remark. The proof below shows that the hypotheses on $\varphi$ may be replaced by the following a priori weaker assumptions: $\varphi$ is a finely harmonic morphism of $U \backslash F$ into $X^{\prime}$ for which there exists a fine component $V$ of $U \backslash F$ such that $\varphi(V)$ is not a polar singleton (in $\left.X^{\prime}\right)$ and further that $C_{f}\left(\varphi_{\mid V}, F\right)$ is a polar subset of $X^{\prime}$. (A similar remark applies to Theorems 4, 6 and 8 below.)

Proof. Let $V$ denote a fine component of $U \backslash F$ with the properties stated in the above remark, and write $V^{\prime}=\varphi(V)$ and $F^{\prime}=C_{f}\left(\varphi_{\mid V}, F\right)$. Note that

$$
E:=U \cap \partial_{f} V \subset U \cap \partial_{f}(U \backslash F) \subset F,
$$

and that $E \neq \emptyset$ (if $F \neq \emptyset$, as we may assume) because $U$ is finely connected.

Consider first the case where $X^{\prime}$ is $\mathfrak{P}$-harmonic, and choose a potential $p^{\prime}$ on $X^{\prime}$ so that $p^{\prime}=+\infty$ on $F^{\prime}$, cf. [8] or [18, Exerc. 6.2.1]. For every $x \in E$ we then have

$$
\text { fine } \lim _{y \rightarrow x, y \in V}\left(p^{\prime} \circ \varphi\right)(y)=+\infty \text {. }
$$


In fact, for any $\lambda \in \mathbf{R}$, the open set $\left\{x^{\prime} \in X^{\prime} \mid p^{\prime}\left(x^{\prime}\right)>\lambda\right\}$ contains $F^{\prime}$ which in turn contains $C_{f}\left(\varphi_{\mid V}, x\right)$ and so there exists a finely open set $W$ containing $x$ such that $\varphi(W \cap V) \subset\left\{x^{\prime} \in X^{\prime} \mid p^{\prime}\left(x^{\prime}\right)>\lambda\right\}$. - Now define

$$
s=\left\{\begin{array}{lll}
p^{\prime} \circ \varphi & \text { in } & V \\
+\infty & \text { in } & U \backslash V .
\end{array}\right.
$$

The above limit relation implies that $s$ is finely continuous, and hence finely hyperharmonic in $U$ according to [26, Lemma 10.1] (cf. also [27, Note 5, p. 82]). Since $V$ is non-polar in $X$, cf. [18, Proposition 6.2.3], $\varphi(V)$ is non-polar in $X^{\prime}$ according to [28, Theorem 6] applied to $\varphi_{\mid V}$, (cf. also [32, § 2.4]), and hence $\varphi(V)$ contains some point at which $p^{\prime}$ is finite. This shows that $\{y \in U \mid s(y)=+\infty\}$ is a proper subset of $U$ and hence polar according to [26, Theorem 12.9], $U$ being a fine domain. In particular, $F(\subset U \backslash V)$ is polar, and hence $U \backslash F$ is finely connected according to [26, Theorem 12.2]. It follows that $U \backslash F=V$ and hence that $C_{f}(\varphi, F)=F^{\prime}\left(\subset X^{\prime}\right)$, and Theorem 1 applies (every component of $X^{\prime}$ being a $\mathfrak{P}$-set).

In the general case, consider again a point $x \in E$, and choose a $\mathfrak{P}$-set $W^{\prime}$ in $X^{\prime}$ containing $K^{\prime}:=C_{f}\left(\varphi_{\mid V}, x\right)$ e.g. as follows: Let $X_{j}^{\prime}$ be any component of $X^{\prime}$. If $X_{j}^{\prime}$ is a $\mathfrak{P}$-set put $W_{j}^{\prime}=X_{j}^{\prime}$. If $X_{j}^{\prime}$ is neither compact nor a $\mathfrak{P}$-set take for $W_{j}^{\prime}$ any relatively compact open set in $X_{j}^{\prime}$ containing the compact set $K^{\prime} \cap X_{j}^{\prime}$. Finally, if $X_{j}^{\prime}$ is compact, but not a $\mathfrak{P}$-set, choose $W_{j}^{\prime}$ as $X_{j}^{\prime}$ less some compact non-polar subset of the open set $X_{j}^{\prime} \backslash K^{\prime}$ (which is non-empty because $K^{\prime}$ is polar). The desired set $W^{\prime}$ is now defined as the union of all the mutually disjoint $\mathfrak{B}$-sets $W_{j}^{\prime}$.

Now let $W$ be a fine domain in $U$ containing $x$ and such that $\overline{\varphi(W \backslash V)} \subset W^{\prime}$. Then $F_{V}:=W \backslash V$ is a relatively finely closed proper subset of $W$ because $W \cap V$ $\neq \emptyset, x$ being on $\partial_{f} V$. The restriction $\psi$ of $\varphi$ to $W \cap V=W \backslash F_{V}$ maps no fine component of $W \cap V$ onto a polar point of $X^{\prime}$, again according to [28, Theorem 6] applied to $\varphi_{\mid V}$. The fine cluster set $C_{f}\left(\psi, F_{V}\right)$ of $\psi$ at $F_{V} \cap \partial_{f}(W \cap V)$ is contained in the $\mathfrak{P}$-set $W^{\prime}$ (because $\overline{\varphi(W \cap V)} \subset W^{\prime}$ ) and also in the polar set $F^{\prime}$ (because $\left.F_{V} \cap \partial_{f}(W \cap V) \subset W \cap \partial_{f} V \subset E \subset F\right)$. Applying what was proved above to the finely harmonic morphism $\psi: W \backslash F_{V} \rightarrow W^{\prime}$ (a $\mathfrak{P}$-set), we conclude that $F_{V}=W \backslash V$ is polar, and that $\psi$, and hence $\varphi_{\mid V}$, has a fine limit at $x$, and therefore at any point of $E$.

Being polar, $W \backslash V$ is thin at $x$, and so is therefore $\complement V$ (because $W$ is a fine neighbourhood of $x$ ). Since $E \subset \complement V$, it follows that $E$ is thin at $x$, and this applies to each $x \in E$, whence $E$ is polar, by Axiom $\mathrm{D}$, cf. [18, Corollary 9.2.3]. This implies that $U \backslash E$ is finely connected, by [25], [26, Theorem 12.2], and since $V \subset U \backslash E$ and $\left(\partial_{f} V\right) \cap(U \backslash E)=\emptyset$, we must have $V=U \backslash E$. But $V \subset U \backslash F \subset U \backslash E$, so that $F=E$, a polar set, and $V=U \backslash F$. Altogether, $\varphi\left(=\varphi_{\mid V}\right)$ has a fine limit at each point of $F(=E)$, and so $\varphi$ admits a finely continuous extension to $U$. Thus Proposition 1 applies, showing that this extension is indeed a finely harmonic morphism. 
The beginning of the above proof in the simple case where $X^{\prime}$ is $\mathfrak{P}$-harmonic carries over mutatis mutandis to establish the following fine topology version of the Riesz-Frostman-Nevanlinna-Tsuji theorem, cf. [79, Theorem VIII.44, p. 339].

Proposition 2. Suppose that $X^{\prime}$ is $\mathfrak{P}$-harmonic. Let $V$ denote a fine domain in $X$, and $E$ a subset of the fine boundary $\partial_{f} V$. Let $\varphi$ be a non-constant finely harmonic morphism of $V$ into $X^{\prime}$. If $C_{f}(\varphi, E)$ is a polar subset of $X^{\prime}$, then $E$ has zero harmonic measure with respect to $V$ in the sense that there exists a finely superharmonic function $s \geqq 0$ on $V$ such that

$$
\text { fine } \lim _{y \rightarrow x} s(y)=+\infty \text { for every } x \in E .
$$

Proof. Write $F^{\prime}:=C_{f}(\varphi, E)$, and proceed as in the very beginning of the proof of Theorem 2. The function $s:=p^{\prime} \circ \varphi$ is seen to have the desired properties.

Remarks. 1) If $X$ is $\mathfrak{P}$-harmonic, the existence of $s$ as stated is equivalent to $E$ being a null set with respect to the (fine) harmonic measure $\varepsilon_{x}^{c V}$ at some (hence any) $x \in V$. This is easily shown by application of results on the (fine) Dirichlet problem for $V$, cf. the end of the proof of Proposition 7, Section 4. Recall that $\varepsilon_{x}^{C V}$ is carried by $\partial_{f} V$ and does not charge the polar sets, cf. [42, Propositions 28.2 et 28.5] or [18, Corollary 7.1.3, Theorem 9.1.1 k), Exerc. 9.2.2 d)].

2) By localizing the notion of sets of zero harmonic measure on $\partial_{f} V$ one can replace the hypothesis that $X^{\prime}$ be $\mathfrak{P}$-harmonic by our standard assumption that $X^{\prime}$ be weakly $\mathfrak{P}$-harmonic. In that case functions like $s$ above will be defined only on $W \cap V$ for a suitable fine neighbourhood $W$ of each point $x \in E$, constructed as in the latter part of the proof of Theorem 2 .

3) Reversing the order of presentation we see that Riesz type results (like Proposition 2) can be used as the first phase in establishing Radó type results (like Theorem 2). This connection has been noticed quite recently by Øksendal [66], [67].

4) In the important case $X=X^{\prime}=\mathbf{C}_{\infty}$, Øksendal [67] has obtained by use of Brownian motion a result stronger than Proposition 2 above. In place of the fine cluster set $C_{f}(\varphi, E)$ he uses the asymptotic set

$$
A(\varphi, E):=\bigcup_{x \in E} A(\varphi, x) \quad\left(\subset \mathbf{C}_{\infty}\right),
$$

where $A(\varphi, x)$ for $x \in \partial_{f} V$ denotes the set of asymptotic values of $\varphi$ at $x$ in the usual sense. (We write $A(\varphi, x)=\emptyset$ if $x$ is inaccessible from $V$.) Note that $A(\varphi, E) \subset$ $C_{f}(\varphi, E)$ because every fine neighbourhood of a point $x \in \mathbf{C}$ contains circular circumferences of arbitrarily small radii, centered at $x$, a well-known property in the plane, not extendable to higher dimensions. Using the Riesz type result thus obtained $\varnothing \mathrm{ksendal}$ deduces a corresponding Radó type result for $\varphi$ a bounded finely harmonic morphism $U \backslash F \rightarrow \mathbf{C}$ (with $U$ a fine domain e.g. in $\mathbf{C}_{\infty}$ ).

In the next result (to be applied in subsequent sections) we need not assume $X^{\prime}$ to be weakly $\mathfrak{P}$-harmonic. On the other hand we impose on $X^{\prime}$ the axiom of 
polarity, cf. $[18, \S 9.1]$. It is a consequence of Axiom $\mathrm{D}$ and hence fulfilled, in particular, by any Riemannian manifold.

Proposition 3. Suppose that $X^{\prime}$ satisfies the axiom of polarity and that the points of $X^{\prime}$ are polar. Let $U$ and $V$ denote finely open subsets of $X$ such that $V \subset U$, and let $\varphi$ be a finely harmonic morphism of $U$ into $X^{\prime}$. If $U \backslash V$ is polar (in $X$ ) then $\varphi(U) \backslash \varphi(V)$ is polar (in $\left.X^{\prime}\right)$.

Proof. We first show that $\varphi(U)$, and similarly $\varphi(V)$, is quasi Borel, i.e., differs only by a polar set from some Borel set. In fact, $U$ has only countably many fine components ([25], [26, §12.1]), and the image of each of these is either a single point of $X^{\prime}$ or else a finely open subset of $X^{\prime}$ according to [28, Theorem 7], [32, $\S 2.4]$; whence the assertion because every finely closed set $A^{\prime} \subset X^{\prime}$ is the union of its base $b\left(A^{\prime}\right)$ and the polar set $A^{\prime} \backslash b\left(A^{\prime}\right)$. (Recall that $b\left(A^{\prime}\right)$ is a $G_{\delta}$ because it intersects each $\mathfrak{P}$-set of $X^{\prime}$ in a $G_{\delta}$, by [18, Corollary 7.2.1], and $X^{\prime}$ is the union of a sequence of $\mathfrak{P}$-sets.)

In proving that the quasi Borel set $\varphi(U) \backslash \varphi(V)$ is polar we may assume that $X^{\prime}$ is $\mathfrak{P}$-harmonic and that $U$ is finely connected. It suffices to prove that any compact subset $K^{\prime}$ of $\varphi(U) \backslash \varphi(V)$ is polar. Proceeding by contradiction suppose that $K^{\prime}$ is non-polar. Choose a finite continuous strict potential $p^{\prime}$ on $X^{\prime}$, cf. [18, $\S 7.2]$, and write $u^{\prime}:=p^{\prime}-\hat{R}_{p^{\prime}}^{K^{\prime}}$. Then $u^{\prime}$ is u.s.c. and $\geqq 0$ on $X^{\prime}$, and superharmonic and $>0$ in $X^{\prime} \backslash K^{\prime}$. Moreover, $u^{\prime}=0$ on the base $b\left(K^{\prime}\right)$, a non-empty subset of the non-polar set $K^{\prime}$; hence $u^{\prime}$ is continuous at each point of $b\left(K^{\prime}\right)$. It follows that $u^{\prime} \circ \varphi \geqq 0$ in $U, u^{\prime} \circ \varphi$ is finely hyperharmonic and $>0$ in $V \subset \varphi^{-1}\left(X^{\prime} \backslash K^{\prime}\right)$, and finely continuous and $=0$ at each point of $\varphi^{-1}\left(b\left(K^{\prime}\right)\right)$, a non-empty subset of the polar set $U \backslash V$. Consequently, the finely hyperharmonic extension of $u^{\prime} \circ \varphi$ from $V$ to $U$, cf. [26, Theorem 9.14], equals 0 on $\varphi^{-1}(b(K))$ in contradiction with [26, Theorem 12.6].

\section{Applications to finely holomorphic and finely meromorphic functions}

First an easy extension to Riemann surfaces of the notion of finely holomorphic function defined on a finely open subset of $\mathbf{C}$ and taking values in C, cf. [28], [20], [21], [55], [56], [33], and the survey [34]. We only consider connected Riemann surfaces (with a countable base).

A Riemann surface $X$ becomes a Brelot harmonic space with a countable base when the sheaf of harmonic functions is taken as the functions which are locally real parts of holomorphic functions from (open subsets of) $X$ into C. Equivalently, the harmonic functions on an open subset of $X$ are the solutions on that set to the Laplace-Beltrami equation with respect to a Riemannian metric on $X$ chosen - as it may be done - so that every holomorphic function (on any open subset of $X$ ) becomes complex harmonic. 
Since the constant functions are harmonic, a Riemann surface $X$ is thus a connected S-Brelot space. It is known that Axiom D holds for every Riemann surface, and that its points are polar. Also recall that $X$ is $\mathfrak{B}$-harmonic if and only if $X$ has a Green function.

Now consider two Riemann surfaces $X, X^{\prime}$. A mapping $\varphi$ of a finely open subset $U$ of $X$ into $X^{\prime}$ is termed finely holomorphic if $\varphi$ is finely continuous (i.e., continuous from $U$ with the fine topology to $X^{\prime}$ with the usual topology) and if moreover $z^{\prime} \circ \varphi \circ z^{-1}$ is finely holomorphic (on the finely open subset $z\left(V \cap \varphi^{-1}\left(V^{\prime}\right)\right.$ ) of $\mathbf{C}$ into $\mathbf{C}$ ) for every choice of complex coordinates $z, z^{\prime}$ on coordinate neighbourhoods $V, V^{\prime}$ in $X, X^{\prime}$, respectively. (Note at this point that the holomorphic bijection $z: V \rightarrow z(V)(\subset \mathbf{C})$ is, in particular, a harmonic isomorphism, hence a homeomorphism in the fine topologies on $V(\subset X)$ and $z(V)(\subset C)$ according to [17, Theorem 3.5].)

Every finely holomorphic mapping $\varphi: U \rightarrow X^{\prime}$ as above is, in particular, a finely harmonic morphism (because $z^{\prime} \circ \varphi \circ z^{-1}$ above is one, cf. [33, §4], [34]). If $U$ is a fine domain and $\varphi$ is non-constant then the pre-image $\varphi^{-1}\left(a^{\prime}\right)$ of any point $a^{\prime} \in X^{\prime}$ is not only polar (equivalently: finely discrete and finely closed), but even countable, cf. [33, §15] for the typical case $X=X^{\prime}=$ C. - Corresponding to Proposition 1 we have

Proposition 4. Let $\varphi$ denote a finely continuous mapping of a finely open subset $U$ of a Riemann surface $X$ into a Riemann surface $X^{\prime}$. If $\varphi$ is finely holomorphic in $U \backslash F$ for some polar set $F$ in $X$ then $\varphi$ is finely holomorphic in all of $U$.

Proof. Via local coordinates $z, z^{\prime}$ for $X, X^{\prime}$ this reduces immediately to the corresponding result for the case $X=X^{\prime}=\mathbf{C}$ which in turn is contained in [33, Cor. 3].

It follows from this proposition that, in the case of Riemann surfaces $X, X^{\prime}$, the results from Section 2 remain valid if the term "finely harmonic morphism" is replaced throughout by "finely holomorphic mapping".

While retaining $X$ as an arbitrary Riemann surface we shall henceforth specialize to $X^{\prime}=\mathbf{C}_{\infty}$, the Riemann sphere. Finely holomorphic mappings of a finely open set $U \subset X$ into $\mathbf{C}$ or $\mathbf{C}_{\infty}$ will be called finely holomorphic functions or finely meromorphic functions, respectively.

Consider a finely meromorphic function $\varphi: U \rightarrow \mathbf{C}_{\infty}$. For any point $a \in U$ and any coordinate $z: V \rightarrow \mathbf{C}$ on $X$ near $a$ such that $z(a)=0$ there is a unique integer $n$ such that $\varphi z^{-n}$ extends by fine continuity to a finely holomorphic function $U \cap V \rightarrow \mathbf{C}$ taking a non-zero value at the given point $a$. (This assertion reduces immediately to the case $X=\mathbf{C}$.) If $\varphi$ itself is finite valued at $a$ (hence in a fine neighbourhood of $a$ ) the existence of this order $n$ (now $\geqq 0$ ) follows from [33, Théorème $11 \mathrm{~b}$ ), Corollaire 3]. If $\varphi(a)=\infty$ then $1 / \varphi: U \cap V \rightarrow \mathbf{C}_{\infty}$ is finely holomorphic off 
the zeros of $\varphi$; and it follows again that $n$ exists as stated (now $n<0$ ), whence $a$ should be called a pole of order $|n|$ for $\varphi$ in this case.

A globally finely meromorphic function is, by definition, a function $\varphi: U \rightarrow \mathbf{C}_{\infty}$ representable as the quotient between two finely holomorphic functions $U \rightarrow$ C. Such a function is, in particular, finely meromorphic. A sufficient condition for a finely meromorphic function $\varphi: U \rightarrow \mathbf{C}_{\infty}$ to be globally finely meromorphic is that $\varphi$ omits at least one value, i.e., $\varphi(U) \neq \mathbf{C}_{\infty}$. [This condition is far from being necessary, as shown e.g. by the (globally) meromorphic function $z \mapsto z+1 / z$ on $\mathbf{C}$ whose range is $\mathbf{C}_{\infty}$.] Another sufficient (but not necessary) condition is that $X$ be non-compact and that $\varphi$ have at most finitely many poles, for then there is a holomorphic function $\psi$ on $X$ such that $\varphi \psi$ becomes finely holomorphic in $U$, cf. e.g. Kra [49, p. 354].

On a usual open subset $U$ of a Riemann surface $X$ the holomorphic and the finely holomorphic functions $U \rightarrow \mathrm{C}$ are the same, cf. [33, p. 63] for the typical case $X=\mathbf{C}$. It follows that the globally meromorphic and the globally finely meromorphic functions on $X$ are the same. (On a non-compact Riemann surface every meromorphic function is globally meromorphic, see $\operatorname{Kra}[49$, p. 355].) - From the above we have the following criterion concerning functions defined on all of $X$ :

Proposition 5. A finely meromorphic function $\varphi: X \rightarrow \mathbf{C}_{\infty}$ is meromorphic if $\varphi(X) \neq \mathbf{C}_{\infty}$, or more generally if every point of $X$ has a (usual) neighbourhood $W$ such that $\varphi(W) \neq \mathbf{C}_{\infty}$.

Example. In $X=\mathrm{C}$ write $z_{n}=2^{-n}$, and choose a sequence of constants $a_{n}$ tending to 0 sufficiently rapidly as $n \rightarrow \infty$ so that $\sum\left|a_{n}\right|<\infty$ and the series $\sum a_{n} /\left(z-z_{n}\right)$ converges uniformly in some fine neighbourhood of 0 . The series converges locally uniformly off 0 and the points $z_{n}$, hence determines altogether a finely meromorphic function $\varphi$ in all of $\mathbf{C}$, cf. [33, p. 74]. (Earlier this example was used by Doob [23, p. 125 f.], and similar examples were studied e.g. by Borel, cf. [6].) Clearly $\varphi$ is not meromorphic in the whole of $\mathbf{C}$, in particular not globally finely meromorphic.

From Theorem 1 combined with Propositions 4 and 3 we immediately obtain the following result of Nevanlinna-Kametani type for finely meromorphic functions:

Theorem 3. Let $U$ denote a finely open subset of a Riemann surface $X$, and $F$ a polar subset of $U$. A finely meromorphic function $\varphi$ on $U \backslash F$ extends to a unique finely meromorphic function $\varphi^{*}$ on $U$ if (and only if) every point of $F$ has a fine neighbourhood $W$ in $U$ such that $\mathbf{C}_{\infty} \backslash \varphi(W \backslash F)$ is non-polar. If even $\mathbf{C}_{\infty} \backslash \varphi(U \backslash F)$ is non-polar then so is $\mathbf{C}_{\infty} \backslash \varphi^{*}(U)$, and hence $\varphi^{*}$ is globally finely meromorphic.

Remarks. 1) This result (for the case $X=\mathbf{C}$ ) was announced in [34]. The typical case where $F$ reduces to a single point $a \in U$ was obtained in conversation with T. J. Lyons and A. G. O'Farrell. - In the case of a usual holomorphic func- 
tion $\varphi: X \backslash\{a\} \rightarrow \mathbf{C}$ (with $X$ non-compact) such that $\mathbf{C} \backslash \varphi(W \backslash\{a\})$ is non-polar for some fine neighbourhood $W$ of $a$ we conclude from Theorem 3 that $\varphi$ extends to a usual meromorphic function in all of $X$ (with $a$ as its only possible pole), viz. to a finely meromorphic function in $X$ with at most one pole. - This result was pointed out by Lyons (in a letter to the author). It is stronger than the otherwise similar result of Casorati-Weierstrass type obtained by Doob [23, Theorem 7.3] (prior to the appearance of finely harmonic or finely holomorphic functions).

2) In view of Propositions 3 and 5 we infer immediately from the first part of Theorem 3 the analogous result in which the word "fine(ly)" is deleted throughout (while $F$ is now a relatively closed polar subset of $U$ ), and we thus recover the result b) stated in the introduction and due essentially to Nevanlinna [61] and Kametani [48]. Alternatively see Theorem 5c in Section 4 below (covering in particular the case of "meromorphic" functions of several complex variables, cf. Section 5). - Note, however, that the example on p. 122 shows that $W$ cannot be allowed to be just a fine neighbourhood in the Nevanlinna-Kametani theorem.

Corollary. Let $F$ denote a polar relatively closed subset of an open set $U$ in $X$. $A$ holomorphic function $\varphi: U \backslash F \rightarrow \mathbf{C}$ extends to a unique holomorphic function on $U$ if (and only if) $C_{f}(\varphi, F) \subset \mathbf{C}$.

Proof. For each $z \in F$ choose a bounded open set $W^{\prime}$ in $\mathbf{C}$ containing the compact set $C_{f}(\varphi, z)$. There is then a fine neighbourhood $W$ of $z$ in $U$ such that $\varphi(W \backslash F) \subset W^{\prime}$. Since $\mathbf{C}_{\infty} \backslash W^{\prime}$ is non-polar, it follows from Theorem 3 that $\varphi$ extends to a unique finely meromorphic function $\varphi^{*}$ on $U$. Since $\varphi^{*}(z) \neq \infty$ also for $z \in F$ (because then $\varphi^{*}(z) \in C_{f}(\varphi, z) \subset \mathbf{C}$ ), $\varphi^{*}$ is finely holomorphic in $U$ and hence holomorphic there.

A further consequence of Theorem 3 involves the irregular part of the boundary of an irregular open set.

Corollary. Let $\varphi$ be meromorphic in a usual open set $V \subset X$ and let $F$ denote the set of irregular points for the Dirichlet problem in $V$. If $\mathbf{C}_{\infty} \backslash \varphi(V)$ is non-polar then $\varphi$ extends by fine continuity to a unique finely meromorphic function $\varphi^{*}$ on the finely open set $V \cup F \subset X$.

Like in Theorem 3 it suffices to suppose that every point of $F$ has a fine neighbourhood $W$ in $X$ such that $\varphi(W \cap V)$ is not co-polar. - Note that, in the affirmative case, very precise information is available as to the behaviour of $\varphi^{*}$ in a suitable fine neighbourhood of each point of $F$, cf. [33, Théorème 11] (for a typical case). - To derive this corollary from Theorem 3, observe that $\varphi$ is, in particular, finely meromorphic in $V=U \backslash F$ with $U:=V \cup F$ finely open, the irregular points for $V$ being precisely the finely isolated points of $\lceil V$.

Next we derive from Theorem 2 a Radó-Stout type theorem for finely meromorphic functions: 
Theorem 4. Let $U$ denote a fine domain in a Riemann surface $X$, and $F$ a relatively finely closed proper subset of $U$. Let $\varphi$ be finely meromorphic in $U \backslash F$ with $\varphi^{\prime} \not \equiv 0$, and suppose that $C_{f}(\varphi, F)$ is polar (in $\mathbf{C}_{\infty}$ ). Then $F$ is polar (in $X$ ), and $\varphi$ extends to a unique finely meromorphic function $\varphi^{*}$ on $U$.

Proof. The hypothesis $\varphi^{\prime} \not \equiv 0$ means that $\varphi$ is not finely locally constant. According to Theorem 2, $F$ is polar, and $\varphi$ extends to a finely harmonic morphism $\varphi^{*}$ of $U$ into $\mathbf{C}_{\infty}$. By Proposition $4, \varphi^{*}$ is finely meromorphic.

For bounded finely holomorphic functions a stronger result has quite recently been obtained by Øksendal [67].

For usual holomorphic functions we obtain the following

Corollary. Let $F$ denote a relatively closed proper subset of a domain $U$ in $X$. If $\varphi$ is holomorphic in $U \backslash F$ with $\varphi^{\prime} \neq \equiv 0$ and if $C_{f}(\varphi, F)$ is polar and contained in $\mathbf{C}$, then $F$ is polar and $\varphi$ extends to a unique holomorphic function in $U$.

In fact, the finely meromorphic extension of $\varphi$ to $U$ omits the value $\infty$ and is therefore holomorphic, by Proposition 5.

Remark. The example on p. 122 shows that one cannot in general, in the meromorphic case, omit the word "fine(ly)" (4 times) in Theorem 4 and still keep the fine cluster set $C_{f}(\varphi, F)$. But if one also replaces the fine cluster set by the usual cluster set then one recovers the Lelong-Stout extension of Radó's theorem mentioned in c) in the introduction. In fact, when $C(\varphi, F)$ is polar, in particular $\neq \mathbf{C}_{\infty}$, then so is the compact set $C(\varphi, z)$ for each point $z$ of $F$ (polar by Theorem 4). Hence $C(\varphi, z)$ has a compact neighbourhood $W^{\prime} \neq \mathbf{C}_{\infty}$, and Proposition 5 applies to a neighbourhood $W$ of $z$ in $U$ such that $\overline{\varphi(W \backslash F)} \subset W^{\prime}$, whence $\varphi^{*}(W) \subset W^{\prime}$. - See also Theorem $6 \mathrm{~b}$ ) below which covers moreover the case of holomorphic (or "meromorphic") functions of several complex variables, cf. Section 5.

\section{Harmonic morphisms}

In this section we shall derive from the results of Section 2 similar results for usual harmonic morphisms, whereby certain subsidiary hypotheses (e.g. $\mathfrak{P}$-harmonicity of the target space $X^{\prime}$ ) are often indispensable. On the other hand, when adapting Theorem 2 and Proposition 2, the hypothesis that the fine cluster set be polar will be weakened (see Theorem 7 and Proposition 7 below).

A harmonic morphism $\varphi: X \rightarrow X^{\prime}$ is, by definition (cf. e.g. [32]), a continuous mapping (with respect to the usual topologies on both spaces) such that $s^{\prime} \circ \varphi$ is hyperharmonic in $\varphi^{-1}\left(V^{\prime}\right)$ for every hyperharmonic function $s^{\prime}$ in an open set $V^{\prime} \subset X^{\prime}$. 
If $X^{\prime}$ has a base of regular sets (e.g., if $X^{\prime}$ is a Brelot space, as in [17]), then this definition is equivalent to the original one in $[17, \S 3]$ in which the word "hyperharmonic" above was replaced by "harmonic" (twice), as noted e.g. in [74], [5], [32].

Every harmonic morphism is likewise a finely harmonic morphism on account of [26, Theorem 8.7]. The key to the application of the results of Section 2 to harmonic morphisms is the following result in the opposite direction.

Proposition 6 (Laine [51]). Suppose that $X^{\prime}$ is a $\mathfrak{P}$-harmonic space and that the points of $X^{\prime}$ are polar. Every finely harmonic morphism $\varphi: X \rightarrow X^{\prime}$ is continuous (in the usual topologies on $X$ and $X^{\prime}$ ) and is therefore a usual harmonic morphism.

Remarks. 1) Let $X^{\prime}$ be a $\mathfrak{P}$-harmonic space satisfying Axiom $\mathrm{D}$ and admitting a bounded harmonic function $h^{\prime}>0$ on $X^{\prime}$. The hypothesis (in Proposition 6) that the points of $X^{\prime}$ be polar can then be weakened to $X^{\prime} \backslash\left\{x^{\prime}\right\}$ being connected for every (non-polar) point $x^{\prime} \in X^{\prime}$. And this condition is always fulfilled if $X^{\prime}$ is a Green space in the sense of Brelot and Choquet [9]. Still more generally it would suffice to assume that $R_{h^{\prime}}^{\left\{x^{\prime}\right.}<h^{\prime}$ in $X^{\backslash} \backslash\left\{x^{\prime}\right\}$ for every non-polar point $x^{\prime} \in X^{\prime}$. This latter observation is obtained by an easy adaptation of the proof in [51]. It covers, e.g., the case where $X^{\prime}$ is a bounded open interval on $\mathbf{R}$ (also covered by [26, Theorem 9.8]).

2) Every finely harmonic morphism $\varphi: X \rightarrow X^{\prime}$ is a harmonic morphism in case $X$ is a 2-dimensional Riemannian manifold, while $X^{\prime}=\mathbf{R}$ or $\mathbf{C}$ or any 2-dimensional Riemannian manifold without compact components. For $X^{\prime}=\mathbf{R}$ or $\mathbf{C}$ this follows at once from the fact that every finely harmonic function on $X$ is harmonic, in particular continuous, so that $\varphi$ must be continuous, see [27]. In the last case we further invoke a result by Greene and Wu [40] according to which every connected non-compact $n$-dimensional Riemannian manifold admits a (proper) embedding in $\mathbf{R}^{2 n+1}$ in terms of harmonic functions on $X$. - The example on p. 122 shows that all this would break down if $X^{\prime}$ were allowed to have compact components, e.g. $X^{\prime}=\mathbf{C}_{\infty}$. On the other hand, the conclusion of Proposition 6 does not hold if $X=\mathbf{R}^{n}$ with $n>2$ and $X^{\prime}=\mathbf{R}$, see the example in [27].

In view of the above proposition we obtain from Theorem 1 the following analogous result for usual harmonic morphisms, keeping in part the fine cluster set.

Theorem 5. Suppose that the points of $X^{\prime}$ are polar. Let $F$ denote a closed polar subset of $X$. A harmonic morphism $\varphi: X \backslash F \rightarrow X^{\prime}$ extends to a unique harmonic morphism of $X$ into $X^{\prime}$ in each of the following 3 situations;

a) $X^{\prime}$ is $\mathfrak{P}$-harmonic and $C_{f}(\varphi, F) \subset X^{\prime}$.

b) $X^{\prime}$ is connected and non-compact, and $C(\varphi, F) \subset X^{\prime}$.

c) $X^{\prime}$ is connected, compact and satisfies the axiom of polarity, and every point of $X$ has a neighbourhood $W$ in $X$ such that $X^{\searrow} \backslash \varphi(W \backslash F)$ is non-polar.

Proof. Ad a): Follows from Theorem 1 in view of Proposition 6. 
Ad b): For any $x \in F$ choose a relatively compact open set $W^{\prime} \subset X^{\prime}$ so that $C(\varphi, x) \subset W^{\prime}$, and next an open set $W \subset X$ so that $x \in W$ and $\overline{\varphi(W \backslash F)} \subset W^{\prime}$, whence $C\left(\varphi_{\mid W \backslash F}, F\right) \subset W^{\prime}$. Since $W^{\prime}$ is a $\mathfrak{P}$-set, it follows from Theorem 1 that $\varphi_{\mid W \backslash F}$ extends from $W \backslash F$ to a finely harmonic morphism of $W$ into $W^{\prime}$, and from Proposition 6 that this extension is a usual harmonic morphism, whence the assertion of the theorem.

Ad c): We may assume that $X^{\prime}$ is not $\mathfrak{P}$-harmonic. By Theorem $1, \varphi$ extends to a finely harmonic morphism $\varphi^{*}: X \rightarrow X^{\prime}$. Taking $W$ open, the open set $W \backslash F$ is a $K_{\sigma}$, and so is $\varphi(W \backslash F)$ because $\varphi$ is continuous. It follows that $X^{\prime} \backslash \varphi(W \backslash F)$ is not even inner polar, and the same applies therefore to $X^{\prime} \backslash \varphi^{*}(W)$ because $\varphi^{*}(W) \backslash \varphi(W \backslash F)$ is polar on account of Proposition 3. Since $X^{\prime}$ is weakly $\mathfrak{P}$-harmonic there exists thereforee a $\mathfrak{P}$-set $W^{\prime}$ of $X^{\prime}$ containing $\varphi^{*}(W)$, and hence the restriction of $\varphi^{*}$ to $W$ is a usual harmonic morphism according to Proposition 6, whence the assertion of the theorem.

Remarks. 1) In the situations described in Remark 2 to Proposition 6, Case b) of Theorem 5 remains in force (in view of Theorem 1 ) when $C(\varphi, F)$ is replaced by $C_{f}(\varphi, F)$. (As an example recall the first corollary to Theorem 3.) In the case $X^{\prime}=\mathbf{R}$ and $F=$ a singleton this is due to Doob [23, Theorem 7.1].

2) For each $x \in F$ the existence of a (usual) neighbourhood $W$ of $x$ such that $\varphi(W \backslash F)$ is not co-polar is equivalent to the range set $\mathfrak{R}(\varphi, x)$ of $\varphi$ at $x$ not being co-polar. Here

$$
\Re(\varphi, x):=\bigcap \varphi(W \backslash F)
$$

where $W$ ranges over a fundamental system (e.g., a countable one) of neighbourhoods of $x$ in $X$. (This is because a countable union of polar sets is polar.)

3) If we combine Theorem 5 above with Theorem 2 in Section 2 we obtain a fine cluster set version of Radó's theorem which may be described as follows: In Theorem 5 we add the hypothesis that $X$ be connected, and replace the assumption that the closed set $F$ be polar (in $X$ ) by the hypothesis that $C_{f}(\varphi, F)$ be a polar subset of $X^{\prime}$ (and that $F \neq X$ ). Assuming moreover that $\varphi$ is not locally constant, we conclude (by Theorem 2) that $F$ is polar, and hence (by Theorem 5) that $\varphi$ extends uniquely to a harmonic morphism of $X$ into $X^{\prime}$. - In the case where $X^{\prime}$ is $\mathfrak{P}$-harmonic this leads to Part a) of the following theorem:

Theorem 6. Suppose that $X$ is connected and that the points of $X^{\prime}$ are polar. Let $F$ denote a closed proper subset of $X$, and $\varphi$ a harmonic morphism, not locally constant, of $X \backslash F$ into $X^{\prime}$. In each of the following situations:

a) $X^{\prime}$ is $\mathfrak{P}$-harmonic and $C_{f}(\varphi, F)$ is a polar subset of $X^{\prime}$, or

b) $C(\varphi, F)$ is a polar subset of $X^{\prime}$, it follows that $F$ is polar (in $X$ ), and that $\varphi$ extends to a unique harmonic morphism of $X$ into $X^{\prime}$. 
Proof of b). Since $C_{f}(\varphi, F) \subset C(\varphi, F)$ it follows from Theorem 2 that $F$ is polar, and that $\varphi$ extends to a unique finely harmonic morphism $\varphi^{*}: X \rightarrow X^{\prime}$. For any $x \in F$ let $Y^{\prime}$ denote the component of $X^{\prime}$ containing the compact connected polar set $C(\varphi, x)$. Choose a relatively compact open set $W^{\prime} \subset Y^{\prime}$ containing $C(\varphi, x)$ and so that $Y^{\prime} \backslash W^{\prime}$ is non-polar (if $Y^{\prime}$ is compact); $W^{\prime}$ is then a $\mathfrak{P}$-set. Finally choose $W$ open in $X$ so that $x \in W$ and $\overline{\varphi(W \backslash F)} \subset W^{\prime}$ and hence $\left(\varphi^{*} W\right) \subset W^{\prime}$. It follows now from Proposition 6 that $\varphi^{*}$, restricted to $W$, is a harmonic morphism of $W$ into $W^{\prime}$, hence also into $X^{\prime}$, from which the assertion follows.

Remarks. 1) Part a) with $C(\varphi, F)$ in place of $C_{f}(\varphi, F)$ is due to Boboc [5] (for slightly more general harmonic spaces $X$ and $X^{\prime}$ than here). See also Oja [65] for further generalizations. - Note that the hypothesis that $X^{\prime}$ be $\mathfrak{P}$-harmonic cannot be removed in a), cf. the example on p. 122.

2) It would be only apparently more general to allow $C(\varphi, F)$ in Theorem 6 b) to be just inner polar (rather than polar). This is because $F$ is a $K_{\sigma}$ in $X$, and so $C(\varphi, F)$ is a $K_{\sigma}$ in $X^{\prime}$. It is easily shown, in fact, for compact $K \subset X$ that

$$
C(\varphi, K)=\bigcap_{W \supset K} \overline{W \text { open }} \overline{\varphi(W)} .
$$

We proceed to partially strengthen Theorem $6 \mathrm{a}$ ) in the spirit of $\varnothing \mathrm{ksendal}$ [66], [67]. For this we assume that $X$ and $X^{\prime}$ are Brelot spaces and that all points of $X^{\prime}$ are strongly polar in the sense of [31], that is, we should have $s^{\prime}\left(x^{\prime}\right)=+\infty$ for every superharmonic function $s^{\prime}$ in some open neighbourhood $U^{\prime}$ of $x^{\prime}$ such that $s^{\prime}$ is harmonic in $U^{\prime} \backslash\left\{x^{\prime}\right\}$, but not at $x^{\prime}$. Under these hypotheses every nonconstant harmonic morphism of a domain $V \subset X$ into $X^{\prime}$ is an open mapping (with respect to the usual topologies on both spaces), see [31]. - Every Riemannian manifold $X^{\prime}$ is a Brelot space, and its points are strongly polar (if $\operatorname{dim} X^{\prime}>1$ ).

Definition 5. Let $V^{\prime}$ be a domain in a $\mathfrak{P}$-harmonic Brelot space $X^{\prime}$. A set $F^{\prime} \subset X^{\prime}$ is said to be polar with respect to $V^{\prime}$ if $F^{\prime} \cap V^{\prime}$ is polar and if $F^{\prime}$ has harmonic measure 0 with respect to $V^{\prime}$ (at some, and hence at any point of $V^{\prime}$ ).

Remark. $V^{\prime}$ is resolutive and has therefore a harmonic measure $\mu_{y^{\prime}}^{V^{\prime}}$ at each point $y^{\prime}$ of $V^{\prime}$, cf. [18, $\S 1.2$ and Theorem 2.4.2]. - It is not difficult to show that $F^{\prime}$ is polar with respect to $V^{\prime}$ if and only if there exists a superharmonic function $s^{\prime} \geqq 0$ on $V^{\prime}$ such that $s^{\prime}=+\infty$ on $F^{\prime} \cap V^{\prime}$ and

$$
\lim _{y^{\prime} \rightarrow x^{\prime}} s^{\prime}\left(y^{\prime}\right)=+\infty \text { at every point } x^{\prime} \in F^{\prime} \cap \partial V^{\prime} .
$$

In particular, a polar set in $X^{\prime}$ is polar with respect to every domain in $X^{\prime}$. (The converse is false when the domain is given.)

Theorem 7. Suppose that $X$ and $X^{\prime}$ are Brelot spaces, that $X$ is connected, that $X^{\prime}$ is $\mathfrak{P}$-harmonic, and that the points of $X^{\prime}$ are strongly polar. Let $F$ denote a 
closed proper subset of $X$. Let $\varphi$ be a harmonic morphism of $X \backslash F$ into $X^{\prime}$, and suppose that there exists a component $V$ of $X \backslash F$ on which $\varphi$ is non-constant and such that $C_{f}\left(\varphi_{\mid V}, F\right) \subset X^{\prime}$ and further that $C_{f}\left(\varphi_{\mid V}, F\right)$ is polar with respect to the domain $\varphi(V)$. Then $F$ is polar, and $\varphi$ extends to a unique harmonic morphism of $X$ into $X^{\prime}$.

Proof. Write $V^{\prime}=\varphi(V)$ and $F^{\prime}=C_{f}\left(\varphi_{\mid V}, F\right)$, and choose $s^{\prime}$ on $V^{\prime}$ as in the remark to Definition 5. Note that $\partial_{f} V \subset \partial_{f}(X \backslash F)(\subset F)$ because $V$ is also a fine component of $X \backslash F$, cf. [26, p. 88]. As in the beginning of the proof of Theorem 2 we then have

$$
\text { fine } \lim _{y \rightarrow x, y \in V}\left(s^{\prime} \circ \varphi\right)(y)=+\infty \text { for every } x \in \partial_{f} V .
$$

In fact, for any $\lambda \in \mathbf{R}, F^{\prime}$ is contained in the interior of $W^{\prime}:=\left\{x^{\prime} \in V^{\prime} \mid s^{\prime}\left(x^{\prime}\right)>\lambda\right\} \cup\left[V^{\prime}\right.$. (Consider separately points of $F^{\prime}$ in $V^{\prime}$, on $\partial V^{\prime}$, or in the interior of $\left[V^{\prime}\right.$.) In particular, $C_{f}\left(\varphi_{\mid V}, x\right) \subset\left(W^{\prime}\right)^{0}$, and so there is a fine neighbourhood $W$ of $x$ in $X$ such that

$$
\varphi(W \cap V) \subset W^{\prime} \cap V^{\prime}=\left\{x^{\prime} \in V^{\prime} \mid s^{\prime}\left(x^{\prime}\right)>\lambda\right\} .
$$

The above limit relation implies that the function $s \geqq 0$ defined by $s=s^{\prime} \circ \varphi$ in $V$ and $s=+\infty$ in $[V$ is finely continuous. Being moreover finely hyperharmonic in $V, s$ is finely hyperharmonic on all of $X$ according to [26, Lemma 10.1], and $s$ is therefore also a usual hyperharmonic function on $X$, cf. [26, Theorem 9.8]. Being open and non-empty, $\varphi(V)$ contains points where $s^{\prime}$ is finite, and so $s \not \equiv+\infty$, showing that $s$ is superharmonic on the connected Brelot space $X$. Since $s=+\infty$ on $F(\subset \complement V), F$ is polar (and $V=X \backslash F$ ), and Theorem 5 a) applies.

The above proof carries over mutatis mutandis to establish the following fine cluster set result of Riesz-Frostman-Nevanlinna-Tsuji type as strengthened by Constantinescu-Cornea [14], [16] and independently by Doob [22], cf. also Øksendal [66], [67]:

Proposition 7. Suppose that $X$ and $X^{\prime}$ are $\mathfrak{P}$-harmonic Brelot spaces and that the points of $X^{\prime}$ are strongly polar. Let $V$ denote a domain in $X$, and $E$ a subset of the fine boundary $\partial_{f} V$. Let $\varphi$ be a non-constant harmonic morphism of $V$ into $X^{\prime}$ such that $C_{f}(\varphi, E) \subset X^{\prime}$. If $C_{f}(\varphi, E)$ is polar with respect to the domain $\varphi(V)$ then $E$ has zero harmonic measure with respect to each point of $V$.

Proof. Write $F^{\prime}:=C_{f}(\varphi, E)$, and proceed as in the proof of Theorem 7, now with $F$ replaced by $E$ and $\varphi_{\mid V}$ by $\varphi$. This leads to a finely superharmonic function $s:=s^{\prime} \circ \varphi \geqq 0$ on $V$ tending finely to $+\infty$ at each point of $E$. Thus $s$ is a "fine superfunction" for the function $f$ equal to $+\infty$ on $E$ and to 0 on the rest of $\partial_{f} V$, cf. [26, §14]. We therefore have $\bar{H}_{f}^{V} \leqq s$, showing that $f$ is integrable with respect to $\varepsilon_{x}^{c V}$ when $x \in V$ and $s(x)<+\infty$. Consequently, $E$ has $\varepsilon_{x}^{c V}$-measure 0 for such $x$, hence actually for any $x \in V$, cf. [26, Corollary 12.6]. 
Remark. Results similar to Proposition 7 are well known for harmonic morphisms in situations where $E$ is a subset of an ideal boundary of a harmonic space $X(=V)$, cf. Constantinescu and Cornea [17], Sibony [76], Ikegami [43], Oja [63], [64]. - These two types of fine cluster set versions of the Riesz-Frostman-Nevanlinna-Tsuji theorem do not seem to imply one another, but unlike the version involving the Martin boundary, say, Proposition 7 does not seem to contain the classical theorem of F. and M. Riesz.

\section{Applications to holomorphic functions of several variables}

Let us specialize the results of Section 4 to holomorphic mappings $X \rightarrow X^{\prime}$, where $X$ is a domain in $\mathbf{C}^{n}, n \geqq 1$, or more generally a Kähler manifold of complex dimension $n$, and where $X^{\prime}$ is $\mathbf{C}$ or $\mathbf{C}_{\infty}$, or just any Riemann surface. Then $X$ and $X^{\prime}$ are connected $\subseteq$-Brelot spaces (hence weakly $\mathfrak{P}$-harmonic) with countable base satisfying Axiom D (in particular the axiom of polarity), and all points of $X$ or $X^{\prime}$ are strongly polar.

Every holomorphic mapping $\varphi: X \rightarrow X^{\prime}$ is a harmonic morphism, cf. [29, p. 114]. It is well known that a continuous mapping $\varphi: X \rightarrow X^{\prime}$ which is holomorphic off some closed polar subset of $X$ is holomorphic in all of $X$, cf. Lelong [54]. This follows also from Theorem 5 in Section 4 according to which $\varphi$ is a harmonic morphism, in particular smooth, and $\varphi$ moreover satisfies locally in $X$ the Cauchy-

Riemann equations $\bar{\partial}_{1} \varphi=\ldots=\bar{\partial}_{n} \varphi=0$ (in terms of local coordinates on $X$ and $X^{\prime}$ ) off $F$, hence actually throughout, by continuity.

With the above circumstances in mind it is clear that the results of Section 4 remain valid for $X$ and $X^{\prime}$ as above if the term "harmonic morphism" is replaced throughout by "holomorphic mapping".

When thus applying Theorems $5 \mathrm{~b}$ and $6 \mathrm{~b}$ in the holomorphic case with $X^{\prime}=\mathbf{C}$, the hypothesis that $C(\varphi, F)$ be a subset of $\mathbf{C}($ i.e., $\infty \notin C(\varphi, F))$ may be replaced by the a priori weaker hypothesis that $\varphi$ be of Hardy class $H^{p}$ (see Definition 6 below) near each point of $F$ for some $p, 0<p<+\infty$. In the case $n=1$ (where $X$ is a Riemann surface) this goes back to Parreau [69], respectively Goldstein and Chow [39] (cf. also Osada [68]). We bring here the $n$-dimensional versions, of which only the Radó type result (Theorem 8 ) involves the fine topology. For simplicity we only state these results in the case where $\varphi$ is globally of class $H^{p}$ (in $X \backslash F$ ).

Definition 6. A holomorphic function $\varphi: U \rightarrow \mathbf{C}(U$ open in $X$ ) is said to be of class $H^{\infty}$ if $\varphi$ is bounded; and of class $H^{p} \quad(0<p<+\infty)$ if the subharmonic function $|\varphi|^{p}$ has a superharmonic majorant (hence also a least harmonic majorant) in $U$.

See Naim [60] for equivalent conditions showing, in particular, that for $n=1$ and $X=\mathbf{C}$ this definition agrees with the classical definition of the Hardy classes $H^{p}$, e.g. on the unit disk. 
Proposition 8. Let $X$ be a domain in $\mathbf{C}^{n}$ (or a Kähler manifold), and let $F$ denote a relatively closed polar subset of $X$. Let $\varphi: X \backslash F \rightarrow \mathbf{C}$ be holomorphic of class $H^{p}$ for some $p, 0<p \leqq+\infty$. Then $\varphi$ extends to a unique holomorphic function on $X$, likewise of class $H^{p}$.

Remark. In the bounded case $p=+\infty$ this is due to Lelong [54], as already mentioned. For $p>1$ the proposition is due to Parreau [69] in the case $n=1$ of a Riemann surface $X$. An alternative proof given by Yamashita [80], consisting in a reduction to the case $p=+\infty$ by application of Gårding and Hörmander's proof [36] of the F. and M. Riesz theorem, carries over to the $n$-dimensional case, as we shall see, using the Fatou-Doob-Naim theorem [59] and the generalization by Naim [60] of the results from [36] on strongly subharmonic functions to the setting of a Brelot harmonic space with its Martin compactification.

Proof. The function $\psi:\left[-\infty,+\infty\left[\rightarrow\left[0,+\infty\left[\right.\right.\right.\right.$ defined by $\psi(t)=e^{p t}$ (interpreted as 0 for $t=-\infty$ ) is positive, increasing, and strictly convex with

$$
\lim _{t \rightarrow+\infty} \frac{\psi(t)}{t}=+\infty
$$

The (pluri)subharmonic function $\log |\varphi|$ on the domain $U:=X \backslash F$ is non-constant because $\varphi$ is an open mapping (if non constant). It follows that

$$
u:=\psi \circ \log |\varphi|=|\varphi|^{p}
$$

is strongly subharmonic (cf. [60, p. 456]) in $U$, but not harmonic. By hypothesis, $u$ has a least harmonic majorant $\hat{u}$ in $U$, and $\hat{u}-u$ is therefore a potential $>0$ on $U$.

Thus $U$ is a $\mathfrak{P}$-Brelot space. Let $\Delta$ denote the corresponding Martin boundary and $\Delta_{1}$ its minimal part. We have then an order preserving bijection $\mu \mapsto K \mu$ carrying the positive Radon measures $\mu$ on $\Delta$ such that $\mu\left(\Delta \backslash \Delta_{1}\right)=0$ onto the harmonic functions $\geqq 0$ on $U$, cf. Herve [42]. Let $m$ denote the measure on $\Delta_{1}$ such that $K m=1$. By the Fatou-Doob-Naim theorem [59] (in the typical case $X \subset \mathbf{C}^{n}$ ) $u$ has a "fine" limit $\breve{u}(x)$ at $m$-almost every point $x$ of $\Delta_{1}$, and $\breve{u} \in L^{1}(m)$. Furthermore we have by [60, Theorem 15]

$$
u=K(\check{u} m)-p
$$

where $p$ is a potential on $U$, and $\check{u} m$ denotes the measure on $\Delta_{1}$ of density $\check{u}$ with respect to $m$. For any natural number $n$ the function

$$
h_{n}:=K(\inf (n, \check{u}) \cdot m)
$$

is bounded ( $\leqq n$ ), harmonic and $\geqq 0$ on $U=X \backslash F$, hence extends to a bounded harmonic function $h_{n}^{*} \geqq 0$ on $X$ (by Bouligand's theorem [7]). The pointwise limit $h^{*}:=\sup _{n} h_{n}^{*}$ coincides in $U$ with the harmonic function $K(\breve{u} m)$ and is therefore 
harmonic in all of $X$. Thus $|\varphi| \leqq\left(h^{*}\right)^{1 / p}$ in $X \backslash F$ with $\left(h^{*}\right)^{1 / p}$ locally bounded in $X$, whence the result according to Lelong's theorem [54].

We proceed to state our $n$-dimensional fine cluster set version of Goldstein and Chow's extension [39] of the Radó-Stout theorem:

Theorem 8. With $X$ as in Proposition 8 let $F$ denote a closed proper subset of $X$, and let $\varphi: X \backslash F \rightarrow \mathbf{C}$ be holomorphic of class $H^{p}$ for some $\left.\left.p \in\right] 0,+\infty\right]$ and not locally constant. If $C_{f}(\varphi, F)$ is polar (in $\mathbf{C}_{\infty}$ ) then $F$ is pluripolar, and $\varphi$ extends to a unique holomorphic function on $X$, likewise of class $H^{p}$.

Remark and proof. In the bounded case $p=+\infty$ this was proved by Cegrell [11], except for our use of the fine cluster set. - To prove Theorem 8 we apply Theorem 2 (taking $X^{\prime}=\mathbf{C}_{\infty}$ ) to show that $F$ is polar. Next Proposition 8 above shows that $\varphi$ has a holomorphic extension $\varphi^{*}$ to $X$ of class $H^{p}$. Since $\varphi^{*}(F)$ $\left(=C_{f}(\varphi, F)\right)$ is polar, it is well known that $F$ must be pluripolar because $s \circ \varphi^{*}$ is plurisubharmonic for every subharmonic function $s$ in a domain of $\mathbf{C}_{\infty}$.

Remarks. 1) Clearly the above results remain valid with the same proofs if the function $s \mapsto s^{p}=\exp (p \log s)$ occuring in Definition 6 is replaced by any function of the form $s \mapsto \psi(\log s)$ with $\psi$ as stated in the beginning of the proof of Proposition 8.

2) In the localized version of Proposition 8 and of Theorem 8, $\varphi: X \backslash F \rightarrow \mathbf{C}$ is just supposed to be of class $H^{p}$ in $W \backslash F$ for some open neighbourhood $W$ of each point of $F$, and the holomorphic extension of $\varphi$ to $X$ is therefore only locally of class $H^{p}$ in general.

3) Since closed sets of $(2 n-2)$-dimensional Hausdorff measure 0 are removable as singularities for (unrestricted) holomorphic functions of $n$ complex variables, the localized version of Theorem 8 can be slightly improved, as noted by Riihentaus [73] in the case $p=+\infty$, by allowing for some set $E \subset F$ of $(2 n-2)$ dimensional Hausdorff measure 0 such that every point just of $F \backslash E$ (rather than of $F$ ) is assumed to have a neighbourhood $W$ as stated in the above remark. (Of course one may here just as well assume that $E$ is closed and that $W \cap E=\emptyset$. It suffices then to apply Theorem 8, now with $X$ replaced by $W$ and $F$ by $F \cap W$ thus showing that $\varphi$ extends holomorphically to each such $W$, hence altogether to $X \backslash E$, and finally automatically to $X$.)

4) Proposition 8 and Theorem 8 can be mixed in the spirit of Cole and Glicksberg [12] (who considered the case $n=1, p=+\infty$ ) so as to yield a simultaneous extension of both. (See also Cegrell [11] and Riihentaus [73] for $n>1, p=+\infty$.) - In our fine cluster set situation this mixture arises from Theorem 8 when the hypothesis that $C_{f}(\varphi, F)$ be polar (in $\left.\mathbf{C}_{\infty}\right)$ is replaced by $C_{f}(\varphi, F \backslash E)$ being polar for some polar set $E \subset F$ (or some pluripolar set $E \subset F$ if we wish to maintain the conclusion that $F$ is pluripolar). Our approach - in which the finely harmonic morphisms are studied first - makes such mixed versions obvious. For 
example, to prove the modified version of Theorem 8 (as just indicated), simply note that Theorem 2 (with $X^{\prime}=\mathbf{C}_{\infty}$ ) applies to $U:=X \backslash E$ (a fine domain) when $F \backslash E$ is used in place of $F$. This shows that $F \backslash E$ is polar, and so is therefore $F$, whence $\varphi$ extends (by Proposition 8) and $F \backslash E$ is even pluripolar (by Theorem 8, or directly).

\section{References}

[1] Aupetit, B.: Une généralisation du théorème d'extension de Radó. - Manuscripta Math. $23: 4,1978,319-323$.

[2] BaIRd, P., and J. Eells: A conservation law for harmonic maps. - Geometry Symposium, Utrecht 1980, 1-25. Lecture Notes in Mathematics 894. Springer-Verlag, BerlinHeidelberg-New York, 1981.

[3] Bernard, A., E. A. Campbell, and A. M. Davie: Brownian motion and generalized analytic and inner functions. - Ann. Inst. Fourier (Grenoble) 29:1, 1979, 207-228.

[4] Besicovitch, A.: On sufficient conditions for a function to be analytic and on behaviour of analytic functions in the neighbourhood of non-isolated singular points. - Proc. London Math. Soc. 32, 1930, 1-9.

[5] Bовос, N.: Asupra prelungirii aplicaţiilor armonice. - An. Univ. Craiova Mat. Fiz.-Chim. 6, 1978, 31-36.

[6] BoREL, É.: Leçons sur les fonctions monogènes uniformes d'une variable complexe. - GauthierVillars, Paris, 1917.

[7] Bouligand, G.: Sur le problème de Dirichlet. - Ann. Soc. Math. Polonaise 4, 1926, 59-112.

[8] Brelot, M.: La convergence des fonctions surharmoniques et des potentiels généralisés. C. R. Acad. Sci. Paris 246, 1958, 2709-2712.

[9] Brelot, M., et G. Choquet: Espaces et lignes de Green. - Ann. Inst. Fourier (Grenoble) 3, 1951, 199-263.

[10] Cartan, H.: Sur une extension d'un théorème de Radó. - Math. Ann. 125, 1952, 49-50.

[11] Cegrell, U.: Generalizations of Radó's theorem. - Preprint. Amer. Math. Soc. Abstracts, Jan. 1981.

[12] Cole, B. J., and I. Glicksberg: Jensen measures and a theorem of Radó. - J. Funct. Anal. $35,1980,26-41$.

[13] Collingwood, E. F., and A. J. Lohwater: The theory of cluster sets. - Cambridge Tracts in Mathematics and Mathematical Physics, No. 56. Cambridge University Press, Cambridge, 1966.

[14] Constantinescu, C., and A. Cornea: Über das Verhalten der analytischen Abbildungen Riemannscher Flächen auf dem idealen Rand von Martin. - Nagoya Math. J. 17, 1960. $1-87$.

[15] Constantinescu, C., and A. Cornea: On the axiomatic of harmonic functions I. - Ann. Inst. Fourier (Grenoble) 13:2, 1963, 373-388.

[16] Constantinescu, C., and A. CoRnea: Ideale Ränder Riemannscher Flächen. - Ergebnisse der Mathematik und ihrer Grenzgebiete, N. F, Bd. 32. Springer-Verlag, Berlin-Göttingen-Heidelberg, 1963.

[17] Constantinescu, C., and A. Cornea: Compactifications of harmonic spaces. - Nagoya Math. J. 25, 1965, 1-57.

[18] Constantinescu, C., and A. Cornea: Potential theory on harmonic spaces. - Die Grundlehren der mathematischen Wissenschaften 158. Springer-Verlag, Berlin-Heidelberg-New York, 1972. 
[19] CSINK, L., and B. ØKSENDAL: Stochastic harmonic morphisms: Functions mapping the paths of one diffusion into the paths of another. - Ann. Inst Fourier (Grenoble) 33:2, 1983, 219--240.

[20] Debiard, A., et B. Gaveau: Potentiel fin et algèbres de fonctions analytiques. I. - J. Funct. Anal. 16, 1974, 289-304.

[21] Debiard, A., et B. Gaveau: Potentiel fin et algèbres de fonctions analytiques. II. - J. Funct. Anal. 17, 1974, 296-310.

[22] Doob, J. L.: Conformally invariant cluster value theory. - Illinois J. Math. 5, 1961, 521549.

[23] Doob, J. L.: Some classical function theory theorems and their modern versions. - Ann. Inst. Fourier (Grenoble) 15:1, 1965, 113-136.

[24] Dоов, J. L.: Applications to analysis of a topological definition of smallness of a set. - Bull. Amer. Math. Soc. 72, 1966, 579-600.

[25] Fuglede, B.: Connexion en topologie fine et balayage des mesures. - Ann. Inst. Fourier (Grenoble) 21:3, 1971, 227-244.

[26] Fuglede, B.: Finely harmonic functions. - Lecture Notes in Mathematics 289. Springer-Verlag, Berlin-Heidelberg-New York, 1972.

[27] Fuglede, B.: Fonctions harmoniques et fonctions finement harmoniques. - Ann. Inst. Fourier (Grenoble) 24:4, 1974, 77-91.

[28] Fuglede, B.: Finely harmonic mappings and finely holomorphic functions. - Ann. Acad. Sci. Fenn. Ser. A I Math. 2, 1976, 113-127.

[29] Fuglede, B.: Harmonic morphisms between Riemannian manifolds. - Ann. Inst. Fourier (Grenoble) $28: 2,1978,107-144$.

[30] Fuglede, B.: Harmonic morphisms. - Proc. Colloq. Complex Analysis Joensuu 1978. Lecture Notes in Mathematics 747. Springer-Verlag, Berlin-Heidelberg-New York, 1979, $123-131$.

[31] Fuglede, B.: Harnack sets and openness of harmonic morphisms. - Math. Ann. 241, 1979, $181-186$.

[32] Fuglede, B.: Invariant characterization of the fine topology in potential theory. - Math. Ann. 241, 1979, 187-192.

[33] Fuglede, B.: Sur les fonctions finement holomorphes. - Ann. Inst. Fourier 31:4, 1981, 57-88.

[34] Fuglede, B.: Fine topology and finely holomorphic functions. - 18th Scandinavian Congress of Mathematicians (Aarhus 1980), Progr. Math. 71, Birkhäuser, Boston Mass. 1981, 22-38.

[35] Fuglede, B.: Value distribution of finely harmonic morphisms and applications in complex analysis. - Potential Theory and its Applications (Kyoto 1983), RIMS Kokyuroku 502. Kyoto 1983, 46-67.

[36] GÅrding, L., and L. Hörmander: Strongly subharmonic functions. - Math. Scand. 15, 1964, 93-96.

[37] Garnetr, J.: Analytic capacity and measure. - Lecture Notes in Mathematics, 297, SpringerVerlag, Barlin-Haidelberg-New York, 1972.

[38] Gehring, F. W., and H. HaAhtr: The transformations which preserve the harmonic functions. - Ann. Acad. Sci. Fenn. Ser. A I Math. 293, 1960, 1-12.

[39] Goldstein, M. G., and T. R. Chow: A theorem of Stout. - Bull. Amer. Math. Soc. 75, 1969, 953-954.

[40] GreENE, R. E., and H. Wu: Embedding of open Riemannian manifolds by harmonic functions. - Ann. Inst. Fourier (Grenoble) 25:1, 1975, 215-235.

[41] HANSEN, W.: Abbildungen harmonischer Räume mit Anwendung auf die Laplace und Wärmeleitungsgleichung. - Ann. Inst. Fourier (Grenoble) 21:3, 1971, 203-216. 
[42] HeRvÉ, R.-M.: Recherches axiomatiques sur la théorie des fonctions surharmoniques et du potentiel. - Ann. Inst. Fourier (Grenoble) 12, 1962, 415-571.

[43] IKEGAMI, T.: Theorems of Riesz type on the boundary behaviour of harmonic maps. - Osaka J. Math. 10, 1973, 247-264.

[44] IKEGAMI, T.: On the boundary behaviour of harmonic maps. - Osaka J. Math. 10, 1973, $641-653$.

[45] Ishinara, T.: A mapping of Riemannian manifolds which preserves harmonic functions. - J. Math. Kyoto Univ. 19:2, 1979, 215-229.

[46] JACOBI, C. G. J.: Über eine particuläre Lösung der partiellen Differentialgleichung $\partial^{2} V / \partial x^{2}+$ $\partial^{2} V / \partial y^{2}+\partial^{2} V / \partial z^{2}=0$. - J. reine u. angew. Math. 36, 1848, 113-134.

[47] JÄRVI, P.: Removability theorems for meromorphic functions. - Ann. Acad. Sci. Fenn. Ser. A I Math. Dissertationes 12, 1977, 1-33.

[48] Kametani, S.: The exceptional values of functions with the set of capacity zero of essential singularities. - Proc. Japan Acad. Tokyo 17, 1941, 429-433.

[49] KrA, I.: Automorphic forms and Kleinian groups. - Mathematics Lecture Note Series. W. Benjamin, Inc., Reading, Mass., 1972.

[50] LaINe, I.: Covering properties of harmonic Bl-mappings. I. - Rev. Roumaine Math. Pures Appl. 19, 1974, 21-34.

[51] LAINe, I.: Covering properties of harmonic $B l$-mappings. II. - Ann. Acad. Sci. Fenn. Ser. A I Math. 570, 1974, 1-14.

[52] LAINe, I.: Covering properties of harmonic Bl-mappings. III. - Ann. Acad. Sci. Fenn. Ser. A I Math. 1, 1975, 309-325.

[53] LAINe, I.: A remark on finely harmonic mappings. - Ann. Acad. Sci. Fenn. Ser. A I Math. 3, 1977, 135-140.

[54] Lelong, P.: Ensembles singuliers impropres des fonctions plurisousharmoniques. - J. Math. Pures Appl. (9) 36, 1957, 263-303.

[55] Lyons, T. J.: Finely holomorphic functions. - J. Funct. Anal. 37, 1980, 1-18.

[56] Lyons, T. J.: A theorem in fine potential theory and applications to finely holomorphic functions. - J. Funct. Anal. 37, 1980, 19-26.

[57 MegheA, C.: Compactification des espaces harmoniques. - Lecture Notes in Mathematics 222. Springer-Verlag, Berlin-Heidelberg-New York, 1971.

[58] MyrberG, P. J.: Über die Existenz der Greenschen Funktionen auf einer gegebenen Riemannschen Fläche. - Acta Math. 61, 1933, 39-79.

[59] NAïM, L.: Sur le rôle de la frontière de R. S. Martin dans la théorie du potentiel. - Ann. Inst. Fourier (Grenoble) 7, 1957, 183-281.

[60] NAïM, L. (=Lumer-Naim, L.): $H^{p}$-spaces of harmonic functions. - Ann. Inst. Fourier (Grenoble) $17: 2,1967,425-469$.

[61] Nevanlinna, R.: Eindeutige analytische Funktionen. - Die Grundlehren der mathematischen Wissenschaften 46. Verlag von Julius Springer, Berlin, 1936 (2. Aufl. 1953).

[62] Noshiro, K.: Cluster sets. - Ergebnisse der Mathematik und ihrer Grenzgebiete. N. F., Heft 28. Springer-Verlag, Berlin-Göttingen-Heidelberg, 1960.

[63] OJA, K.: On cluster sets of harmonic morphisms between harmonic spaces. - Ann. Acad. Sci. Fenn. Ser. A I Math. Dissertationes, 24, 1979, 1-51.

[64] OJA, K.: Theorems of the Riesz type for co-fine cluster sets of harmonic morphisms. - Ann. Acad. Sci. Fenn. Ser. A I Math. 6, 1981, 77-88.

[65] OJA, K.: Exceptional sets and harmonic morphisms. - Math. Ann. 262:1, 1983, 45-56.

[66] ØKsendal, B.: A stochastic proof of an extension of a theorem of Radó. - Proc. Edinburgh Math. Soc. (2) 26, 1983, 333-336.

[67] ØKSENDAL, B.: Finely harmonic morphisms, Brownian path preserving functions and conformal martingales. - Invent. Math. 75, 1984, 179-187. 
[68] OsADA, M.: A generalization of a theorem of Radó-Stout to analytic functions on open Riemann surfaces. - J. Hokkaido Univ. Ed. Section IIA 29, 1, 1978, 1-3.

[69] Parreau, M.: Sur les moyennes des fonctions harmoniques et analytiques et la classification des surfaces de Riemann. - Ann. Inst. Fourier (Grenoble) 3, 1951, 103-197.

[70] Pirinen, A.: Absorbent sets and harmonic morphisms. - Ann. Acad. Sci. Fenn. Ser. A I Math. Dissertationes 42, 1982, 1-49.

[71] Popa, E.: Finely open morphisms of $H$-cones. - Math. Ann. (to appear).

[72] RADó, T.: Über eine nicht fortsetzbare Riemannsche Mannigfaltigkeit. - Math. Z. 20, 1924, $1-6$.

[73] Riıhentaus, J.: A note concerning Radó's theorem. - Math. Z. 182, 1983, 159-165.

[74] SCHIRMEIER, U.: Isomorphie harmonischer Räume. - Math. Ann. 225, 1977, 33-55.

[75] SCHirmeiER, U.: Banach-Stone-type theorems for harmonic spaces. - Romanian-Finnish Seminar on Complex Analysis (Bucharest 1976). Lecture Notes in Mathematics 743, Springer-Verlag, Berlin-Heidelberg-New York, 1979, 573-580.

[76] Sibony, D.: Allure à la frontière minimale d'une classe de transformations. Théorème de Doob généralisé. - Ann. Inst. Fourier (Grenoble) 18:2, 1968, 91-120.

[77] Stout, E. L.: A generalization of a theorem of Radó. - Math. Ann. 177, 1968, 339-340.

[78] SzEGŐ, G.: Bemerkungen zu einer Arbeit von Herrn M. Fekete: Über die Verteilung der Wurzeln bei gewissen algebraischen Gleichungen mit ganzzahligen Koeffizienten. Math. Z. 21, 1924, 203-208.

[79] Tsuji, M.: Potential theory in modern function theory. - Maruzen 10., Ltd., Tokyo, 1959.

[80] Yamashita, S.: On some families of analytic functions on Riemann surfaces. - Nagoya Math. J. 31, 1968, 57-68.

[81] ZoRETTI, L.: Sur les fonctions analytiques uniformes qui possèdent un ensemble parfait discontinu de points singuliers. - J. Math. Pures Appl. 6, 1905, 1-51.

\author{
University of Copenhagen \\ Institute of Mathematics \\ DK-2100 København \\ Denmark
}

Received 27 July 1984 